\title{
Knickung von Schraubenfedern unter Druck und konservativer Torsion
}

\section{Doctoral Thesis}

Author(s):

Maag, Heinrich

Publication date:

1957

Permanent link:

https://doi.org/10.3929/ethz-a-000096550

Rights / license:

In Copyright - Non-Commercial Use Permitted 


\title{
Diss ETH
}

\section{KNICKUNG VON \\ SCHRAUBENFEDERN UNTER DRUCK UND KONSERVATIVER TORSION}

VON DER

\section{EIDGENÖSSISCHEN TECHNISCHEN HOCHSCHULE IN ZÜRICH \\ ZUR ERLANGUNG}

DER WÜRDE EINES DOKTORS DER MATHEMATIK

GENEHMIGTE

PROMOTIONSARBEIT

\author{
VORGELEGT VON \\ HEINRICH MAAG \\ VON ZÜRICH UND OBERGLATT/ZH
}

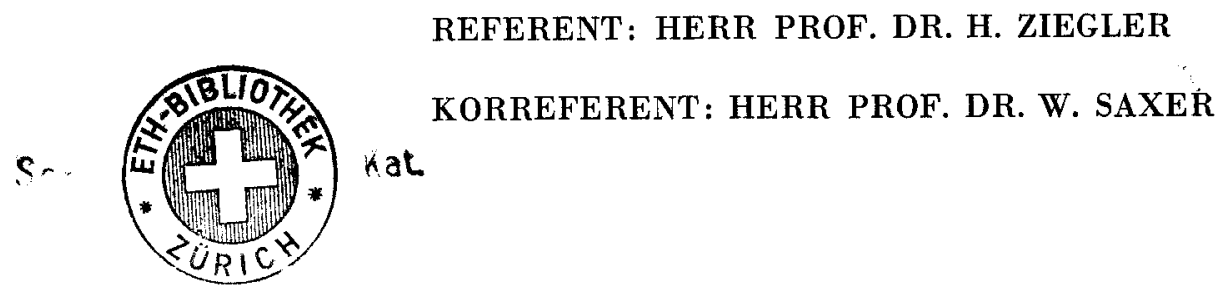

SPRINGER-VERLAG · BERLIN / GÖTTINGEN / HEIDELBERG 


\title{
Knickung von Schraubenfedern unter Druck und konservativer Torsion
}

\author{
Von H. Maag
}

1. Einleitung. Die Knicklasten einer durch eine axiale Druckkraft beanspruchten Schraubenfeder wurden erstmals von E. Hurlbrink ${ }^{1}$ und in einem allgemeineren Rahmen 1924 von R. Grammel ${ }^{2}$ berechnet, indem die Feder in geeigneter Weise durch einen weichen Stab ersetzt wurde. C. B. Biezeno und J. J. Koch ${ }^{3}$ zeigten, daß die Resultate durch Berücksichtigung des Einflusses der Querkraft speziell für nichtschlanke Federn erheblich verbessert werden können, und $J$. A. Haring $x^{4,5}$ machte neuerdings darauf aufmerksam, daß man dann auch die von der Querkraft erzeugte Schiebung berücksichtigen muß. In dieser Arbeit berechnete Haringx die kritischen Lasten für eine Belastung, die sich aus einer axialen Druckkraft und einem axialen Torsionsmoment zusammensetzt.

Diese Resultate wurden alle mit Hilfe des statischen Stabilitätskriteriums gefunden, wonach die Stabilitätsgrenze durch das erstmalige Auftreten von nichttrivialen, d. h. ausgebogenen Gleichgewichtslagen bedingt ist. Nun hat aber H. Ziegler ${ }^{6}$ gezeigt, daß das statische Stabilitätskriterium nur für konservative Systeme legitim ist, also insbesondere auf Systeme, in denen axiale Torsionsmomente auftreten, nicht angewendet werden darf, da diese ja im allgemeinen nicht konservativ sind. Die Resultate von Haringx hängen damit teilweise in der Luft und bedürften einer Überprüfung durch das kinetische Stabilitätskriterium (Untersuchung kleiner Schwingungen um die Gleichgewichtslage), das in allen Fällen gültig ist.

Anderseits stellte H. Ziegler ${ }^{7}$, indem er auf die den Momentvektor erzeugenden Einzelkräfte zurückging, fest, daß die durch die einfachsten Kraftverteilungen erzeugten Momentvektoren nur bei Einspannungen axial bleiben, bei gelenkig gelagerten oder freien Enden aber ihre Richtung ändern. Die so je nach dem Kraftangriff entstehenden semi-, quasi- und pseudotangentialen Momente sind alle konservativ. Für die Belastung mit solchen Momenten darf also wieder das statische oder auch das energetische Stabilitäts. kriterium angewendet werden, wonach die Stabili-

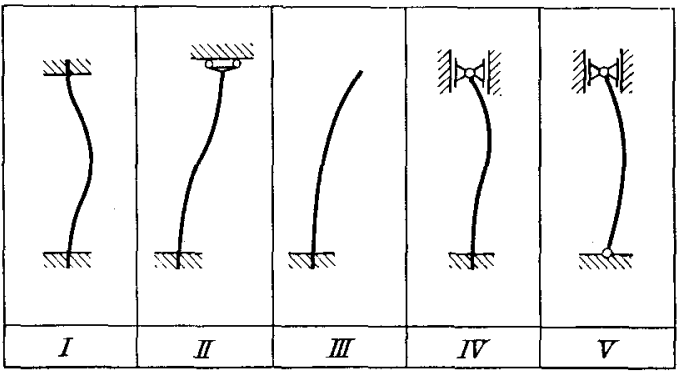

Abb. 1. Die untersuchten Lagerungen.

I. die beidseitig horizontal unverschieblich eingespannte Feder, II. die beidseitig eingespannte Feder mit gegeneinander horizontal verschiebbaren Einspannungen,

IV. die auf der einen Seite eingespannte, auf der andern gelenkig

v. die beidseitig gelenkig gelagerte Feder.

tätsgrenze erreicht ist, wenn die potentielle Energie desSystemes erstmals nicht mehr positiv definit ist.

In der vorliegenden Arbeit wird nun die Knickung einer Feder untersucht, deren Belastung sich aus einer axialen Druckkraft und einem solchen konservativen Torsionsmoment zusammensetzt. Im Abschnitt 2 werden die Differentialgleichungen der elastischen Linie der Feder bzw. des elastischäquivalenten Ersatzstabes hergeleitet. Unter Berücksichtigung der Randbedingungen werden dann in Abschnitt 3 die Knickgleichungen aufgestellt und zwar für fünf Lagerungsarten (Abb. 1).

Selbstverständlich müssen in allen fünf Fällen die Lager so ausgebildet sein, daß sich die Federenden gegeneinander um die ursprüngliche Federachse verdrehen und vertikal verschieben können. Im Abschnitt 4 schließlich werden die Knickgleichungen diskutiert und in den einfacheren Fällen die kritischen Dynamen in Diagrammen dargestellt.

2. Grundlagen. a) Der Ers at z s ta b. Eine Schraubenfeder, deren Länge im ungespannten Zustand $l_{0}$ und deren Wicklungsradius $R$ beträgt, sei durch die Belastung auf die Länge $l$ zusammengedrückt. Die Feder sei so gewickelt, daß die Hauptträgheitsachsen des Drahtquerschnittes parallel

1 E. Hurlbrink, Z. VDI, 54 (1910) S. 133 u. 181.

${ }^{2}$ R. Grammel, Z. angew. Math. Mech. 4 (1924) S. 384.

${ }^{3}$ C. B. Biezeno u. J. J. Koch, Z. angew. Math. Mech. 5 (1925) S. 379.

4 J. A. Haringx, Proc. Nederl Akad. Wet. 45 (1942) S. 533 u. 650.

5 J. A. Haringx, Philips Res. Rep. 3 (1948) S. 401 u. 4 (1949) S. 49.

${ }^{6} H$. Ziegler, Z. angew. Math. Phys. 4 (1953) S. 89.

7 H. Ziegler, Z, angew. Math. Phys. 3 (1952) S. 96. 